\title{
ASYMPTOTIC AND NUMERICAL SOLUTIONS FOR DIFFUSION MODELS FOR COMPOUNDED RISK RESERVES WITH DIVIDEND PAYMENTS
}

\author{
S. SHAO and C. L. CHANG
}

Received 17 April 2003

\begin{abstract}
We study a family of diffusion models for compounded risk reserves which account for the investment income earned and for the inflation experienced on claim amounts. We are interested in the models in which the dividend payments are paid from the risk reserves. After defining the process of conditional probability in finite time, martingale theory turns the nonlinear stochastic differential equation to a special class of boundary value problems defined by a parabolic equation with a nonsmooth coefficient of the convection term. Based on the behavior of the total income flow, asymptotic and numerical methods are used to solve the special class of diffusion equations which govern the conditional ruin probability over finite time.
\end{abstract}

2000 Mathematics Subject Classification: 91B30, 35K20, 35B40, 65M06.

1. Introduction. A risk reserve is set up to provide financial support in the event of a loss for the collection of policy holders. The diffusion-type modelling for risk reserves is one of the most popular methods for the valuing of the investment income earned and for the inflation experienced on claim amounts in studying insurance models. The basic assumption is that the risk reserve is modelled as a one-dimensional diffusion process. In a continuous time aspect of the system under study, diffusion process is an arbitrary strong Markov process with continuous sample paths, or even more specifically, diffusion process is given as a strong solution of a stochastic differential equation driven by the underlying Brownian motion. This approach, which uses an appropriate diffusion process to approximate the actual claims process, has been examined by many authors during the past twenty years including Garrido [4, 5], Young and Zariphopoulou [13], Shao [10], and many others. Garrido [5] studied a family of diffusion models for compounded risk reserves. For the linear case, he obtained an explicit solution of the stochastic differential equation for the distribution of the time to ruin. For the nondegenerate diffusion processes of risk, Young and Zariphopoulou [13] provided an analysis for distorted survival probabilities related to risks in incomplete markets. Shao [10] studied the asymptotic behavior of a family of diffusion models for risk reserves with the situation of no dividend payments. By allowing possibly "degenerates" of the diffusion equation, Shao provided the asymptotic solution of the probability of ruin over finite time. In this paper, we study the extended models of [10] to treat a more complicated diffusion model for compounded risk reserves, which allow the dividend payments to be paid from the risk reserves. The main tools come from 
the theories of diffusion process, partial differential equations, asymptotic analysis, numerical methods, and applied probabilities.

Consider the risk reserves accumulated for a particular line of business. Then diffusion process $R(t)$ is the stochastic dynamics of the size of the risk reserve level at time $t$. Let $\pi(t, R(t))$ be the aggregate rate at which premiums are cashed at time $t$, let $\mu(t)$ be the average aggregated claim rate (average accumulated claim rate) at time $t$, and let $\beta(t)$ be the assumed known force of reserve earning interest rate at time $t$. Then given the initial risk reserve $r_{0}$, the well-known dynamics of the risk reserve is governed by the nonlinear stochastic differential equations

$$
\begin{gathered}
d R(t)=\gamma(t, R(t)) d t+\sigma(t, R(t)) d W(t), \\
R(0)=r_{0} \geq 0,
\end{gathered}
$$

where $\gamma(t, R(t))=[\pi(t, R(t))+\beta(t) R(t)-\mu(t)]$ is the total income dynamics, $W(t)$ is a standard Brownian motion defined on the probability space $\left(\Omega, F_{t}, P\right)$ with $F_{t}$ the augmentation of the $\sigma$-algebra generated by the realizations of the Brownian motion up to time $t$ and with $P$ a probability measure on $\left(\Omega, F_{t}\right)$, and $\sigma(t, R(t))$ is the volatility coefficient which is randomly fluctuating according to mean-reverting diffusion process. We also assume that $\beta(t), \mu(t)$, and $\sigma(t, R(t))$ are functions of class $C^{2}$ within the given domain and that $\gamma(t, R(t))$ and $\sigma(t, R(t))$ satisfy the usual growth and Lipschitz conditions

$$
\begin{gathered}
|\gamma(t, x)-\gamma(t, y)|+|\sigma(t, x)-\sigma(t, y)| \leq M|x-y|, \\
|\gamma(t, x)|^{2}+|\sigma(t, x)|^{2} \leq M(1+|x|)^{2},
\end{gathered}
$$

for some positive constant $M$. These two conditions guarantee that a unique solution to (1.1) exists (see [6, Chapter 6]).

We note that $\pi(t, R(t))$ depending on the current value of the reserve at time $t$ serves to model the collection of premiums rate. For example, when $\pi(t, R(t))=\pi_{0}$, where $\pi_{0}$ is a constant, the total income dynamics $\gamma(t, R(t))=\pi_{0}+\beta(t) R(t)-\mu(t)$ serves to model the idle reserve earning interest at a rate $\beta(t)$ with the average aggregated claim rate $\mu(t)$, which is the simplest case. If we allow the dividend payments to be paid from the risk reserves, then the premium rate $\pi(t, R(t))$ varies with the reserve level. In practice, these are usually made at the end of the policy term and take the form of a partial reimbursement of the charged premium. Hence we assume a continuous payment of premiums and let these dividend payments be made continuously. In general, large reserves will prompt dividend payments and a reimbursement of a portion of the collected premium. Therefore, the premium rate $\pi(t, R(t))$ can be defined by

$$
\pi(t, R(t))= \begin{cases}\pi(t), & \text { if } R(t) \leq d_{1}(t), \\ \pi(t)\left(1-\rho\left[\frac{R(t)-d_{1}(t)}{d_{2}(t)-d_{1}(t)}\right]\right), & \text { if } d_{1}(t)<R(t) \leq d_{2}(t) \\ \pi(t)(1-\rho), & \text { if } d_{2}(t)<R(t),\end{cases}
$$


where $d_{i}(t)$ is a dividend barrier at time $t$, for $i=1,2$, and $\rho$ is the portion of the premium returned when reserves are larger than the largest dividend barrier. We notice that this premium rate $\pi(t, R(t))$ is continuous and interpolates between the no-dividend situation when reserves are low $(\pi(t, R(t))=\pi(t))$ and the full-dividend cases where reserves are high $(\pi(t, R(t))=\pi(t)(1-\rho))$. Moreover, the drift term mapping

$$
x \longrightarrow \gamma(t, x)=\pi(t, x)+\beta(t) x-\mu(t)
$$

is continuous. Most of the information can be found in [5, 12].

Our first effort is to show that the diffusion models for compounded risk reserves with dividend payments (1.1) and (1.3) can be solved by an initial boundary value problem defined by a parabolic equation

$$
\begin{gathered}
\frac{\partial \phi}{\partial t}+\gamma(t, r) \frac{\partial \phi}{\partial r}+\frac{1}{2} \sigma^{2}(t, r) \frac{\partial^{2} \phi}{\partial r^{2}}=0, \\
\phi(t, r)=1, \quad r<0, \quad \phi(T, r)=0, \quad r>0,
\end{gathered}
$$

which governs the conditional probability of ruin $\phi(t, r)$ occurring between times $t$ and $T$, given the reserve $r$ at time $t$, where $\sigma(t, r)$ is the volatility coefficient (diffusion coefficient) and $\gamma(t, r)$ is defined by

$$
\gamma(t, r)= \begin{cases}\pi(t)+\beta(t) r-\mu(t), & \text { if } r \leq d_{1}(t), \\ \pi(t)\left(1-\rho\left[\frac{r-d_{1}(t)}{d_{2}(t)-d_{1}(t)}\right]\right)+\beta(t) r-\mu(t), & \text { if } d_{1}(t)<r \leq d_{2}(t), \\ \pi(t)(1-\rho)+\beta(t) r-\mu(t), & \text { if } d_{2}(t)<r,\end{cases}
$$

for $0 \leq t \leq T<\infty$.

This approach is particularly important because although the study of partial differential equations in complete generality is a vast undertaking, almost all the partial differential equations encountered in financial applications belong to a much more manageable subset of the whole second-order parabolic equations. The important studies of diffusion processes and their connection with parabolic partial differential equations were given by Freidlin [3], and Fleming and Soner [2]. Their studies in the past twenty years presented thorough results on the subject of viscosity solutions. Our focus is to show that the classical results from the theory of diffusion processes and martingales can be applied to (1.1) and (1.3) to yield a special class of boundary value problems defined by a parabolic equation (1.5).

Our second effort is to provide solutions to that special class of boundary value problem of (1.5) and (1.6) of diffusion equations. We note that $\gamma(t, r)$ defined in (1.6) is continuous but not smooth (it is not differentiable). It is unlikely that (1.5) and (1.6) can be solved analytically because of the nonsmoothness of the coefficient of the convection term $\gamma(t, r)$. Moreover, the boundary condition in (1.5) is defined on the left half-opened space $r<0$. In fact, the classical solutions of (1.5) and (1.6) may not exist since the differential operator is possibly degenerate when volatility is in fast mean reverting. Our focus in this part is to obtain asymptotic numerical solutions of (1.5) and (1.6) by using asymptotic analysis to guide the numerical solutions, which illustrates the behavior of 
the conditional probability of ruin $\phi(t, r)$ over finite-time horizon. It is well known that numerical solutions of boundary value problems of partial differential equations often use a variety of finite-difference or finite-element formulations of differential equations. We use finite-difference methods to obtain numerical solutions of (1.5) when volatility coefficient (diffusion coefficient) $\sigma$ is not "small." When $\sigma \ll 1$, that is, in the presence of a separation of time scales, between the main observed process and the volatility driving process, regular finite-difference or finite-element methods fail in this situation. However, asymptotic methods are very efficient in capturing the effects of random volatility in simple robust corrections to constant volatility. From the point of view of partial differential equations, this method corresponds to a singular perturbation analysis. An asymptotic-numerical method will be used to obtain solutions of (1.5). Our aim is to understand how the rate of the reserve growth $\gamma(t, r)$ and dividend payments affect the behavior of the conditional probability of ruin at a given reserve level $r$. The asymptotic behavior of the solutions is analyzed for three fundamental regions according to the signs of $\gamma(t, r)$. Numerical illustrations given to show some of our numerical results will also be compared with the results in [10] obtained from the asymptotic approximations.

In Section 2, we state some basic definitions and results from stochastic calculus. Then we show that the diffusion models for compounded risk reserves (1.1) can be solved by a boundary value problem defined by a special class of a parabolic equation. One internal layer theorem from singular perturbation theory is introduced before we close Section 2. In Section 3 the numerical and asymptotic analysis to be used to obtain the approximate solutions for different income flow is presented. Examples are provided to illustrate the solution behavior of the conditional probability of ruin over finite time. The final conclusion and remarks will be discussed in Section 4.

2. Diffusion models and parabolic partial differential equations. In this section, we start with a brief review of the fundamental results of the theory of stochastic calculus and martingale theory $[1,7,8,9]$, then we impose appropriate boundary conditions and derive boundary value problems of the parabolic partial differential equations from the diffusion models for compounded risk reserves (1.1).

DEFINITION 2.1. Let $R(t)$ be a risk reserve process that is a model for the time evolution of the reserves at time $t$, let the initial reserve $R(0)=r_{0} \geq 0$, and let $F_{t}=$ $\sigma\{W(s): 0 \leq s \leq t\}$ be the $\sigma$-field generated by a standard Brownian motion $W(t)$.

(a) The conditional probability $\phi(t, r)$ defined by

$$
\phi(t, r)=P\left(\inf _{t \leq s \leq T} R(s)<0 \mid R(t)=r\right)
$$

is called the probability of ruin between $t$ and $T$ with the given reserve $r$ at time $t$. In other words, the conditional probability of ruin is the probability that reserve ever drops below zero between $t$ and $T$ with the given reserve $r$ at time $t$.

We notice that ruin could happen, for instance, if the company pays out pensions or if it invests its reserve and gets a negative outcome of its investments. By definition (a), the probability of ruin is 1 when the reserve becomes negative; the initial probability 
of ruin is 0 at time $t=T$ when the reserve is positive. Therefore, we obtain the initial boundary conditions

$$
\phi(t, r)=1, \quad r<0, \quad \lim _{r \rightarrow 0} \phi(t, \tau)=1, \quad \phi(T, r)=0, \quad r>0,
$$

with $0 \leq t<T \leq \infty$.

(b) The time $\tau$ defined by

$$
\tau=\inf \{t \geq 0 \mid R(t)<0\}
$$

is the first time at which the reserve level becomes negative and it is called the time of ruin.

(c) The first dividend payment time

$$
T^{d}=\inf \{t<0 \mid R(t)>d(t)\}
$$

is defined by reserves $R(t)$ reaching a preassigned dividend barrier $d(t)$ for the first time.

(d) A process $S$ is called a martingale with respect to a probability measure $P$ and a family of $\sigma$-field $F_{i}$ generated by the process $S$ if

$$
E_{P}\left(S_{j} \mid F_{i}\right)=S_{i} \quad \forall i \leq j .
$$

THEOREM 2.2. Suppose that $g$ is a function of class $C^{2}\left(R^{k}, R\right)$ and that the processes $X^{i}$ are in the class of all real-valued continuous semimartingales on the probability space for $i=1,2, \ldots, k$, then the following form of Ito's formula is valid:

$$
g(X)-g\left(X_{0}\right)=\sum_{i=1}^{k} \int_{0}^{t} \frac{\partial g}{\partial x_{i}}\left(X_{u}\right) d X_{u}^{i}+\frac{1}{2} \sum_{i, j=1}^{k} \int_{0}^{t} \frac{\partial^{2} g}{\partial x_{i} x_{j}}\left(X_{u}\right) d\left(\left\langle X^{i}, X^{j}\right\rangle_{u}\right) .
$$

Now we have the following theorem.

THEOREM 2.3. If $\phi(t \wedge \tau, R(t \wedge \tau))$ is an $F_{t \wedge \tau}$ martingale, that is,

$$
\phi(t \wedge \tau, R(t \wedge \tau))=E\left(I(\tau<T) \mid F_{t \wedge \tau}\right)
$$

where $t \wedge \tau=\min \{t, \tau\}$, then the conditional probability of ruin $\phi(t, r)$ satisfies the parabolic equation

$$
\frac{\partial \phi}{\partial t}+\gamma(t, r) \frac{\partial \phi}{\partial r}+\frac{1}{2} \sigma^{2}(t, r) \frac{\partial^{2} \phi}{\partial r^{2}}=0
$$

subject to the initial boundary conditions (2.2), where $\gamma(t, r)$ is as defined in (1.6).

Proof. Consider the stochastic dynamics of the size of the risk reserve level at time $t$ in (1.1),

$$
\begin{gathered}
d R(t)=\left[\pi(t, R(t))+\beta_{t} R(t)-\mu(t)\right] d t+\sigma(t, R(t)) d W(t), \\
R(0)=r_{0} \geq 0,
\end{gathered}
$$


with the premium rate $\pi(t, R(t))$ defined by (1.3). Recall that $W(t)$ is a standard Brownian motion, $r$ is the reserve level, $\pi(t, R(t))$ is the aggregate rate at which premiums are cashed at time t, $\mu(t)$ is the average aggregated claim rate at time $t, \beta(t)$ is assumed known force of reserve earning interest rate at time $t$, and $d_{i}(t)$ is a dividend barrier at time $t$, for $i=1,2$. The drift term mapping $x \rightarrow \gamma(t, x)=\pi(t, x)+\beta(t) x-\mu(t)$ is continuous. Since the coefficients of the stochastic differential equations (1.1) depend only on the history through $R(t)$, therefore the risk reserve process $R(t)$ is a Markov process (recall that Markov process is a stochastic process where the behavior of the variable over a short period of time depends solely on the value of the variable at the beginning of the period, not on its past history). Hence, the conditional probability of ruin

$$
\begin{aligned}
\phi(t \wedge \tau, R(t \wedge \tau)) & =P\left(\inf _{t \wedge \tau \leq s \leq T} R(s)<0 \mid R(t \wedge \tau)\right) \\
& =E\left(I\left(\inf _{t \wedge \tau \leq s \leq T} R(s)<0\right) \mid R(t \wedge \tau)\right) \\
& =E(I(\tau<T) \mid R(t \wedge \tau)) \\
& =E\left(I(\tau<T) \mid F_{t \wedge \tau}\right)
\end{aligned}
$$

shows that the strong Markovian property of $R(t)$ implies that $\phi(t \wedge \tau, R(t \wedge \tau))$ is an $F_{t \wedge \tau}$ martingale. Thus $\phi(t \wedge \tau, R(t \wedge \tau))-\phi_{0}\left(0, r_{0}\right)$ is a zero initial valued martingale and it is square integrable. Applying Ito's formula of Theorem 2.2, we have that

$$
\begin{aligned}
\phi(t \wedge & \tau, R(t \wedge \tau))-\phi_{0}\left(0, r_{0}\right) \\
= & \int_{0}^{t \wedge \tau} \frac{\partial \phi}{\partial s}(s, r) d s+\int_{0}^{t \wedge \tau} \frac{\partial \phi}{\partial r}(s, r) d R \\
& +\frac{1}{2} \int_{0}^{t \wedge \tau} \frac{\partial^{2} \phi}{\partial r^{2}}(s, r) \sigma^{2}(s, r) d s \\
= & \int_{0}^{t \wedge \tau} \frac{\partial \phi}{\partial s}(s, r) d s+\int_{0}^{t \wedge \tau} \frac{\partial \phi}{\partial r}(s, r)[\pi(t, r)+\beta(t) r-\mu(t)] d s \\
& +\int_{0}^{t \wedge \tau} \frac{\partial \phi}{\partial r}(s, r) \sigma(s, r) d W(t)+\frac{1}{2} \int_{0}^{t \wedge \tau} \frac{\partial^{2} \phi}{\partial r^{2}}(s, r) \sigma^{2}(s, r) d s
\end{aligned}
$$

implies

$$
\begin{gathered}
\int_{0}^{t \wedge \tau} \frac{\partial \phi}{\partial s}(s, r) d s+\int_{0}^{t \wedge \tau} \frac{\partial \phi}{\partial r}(s, r)[\pi(t, r)+\beta(t) r-\mu(t)] d s \\
+\frac{1}{2} \int_{0}^{t \wedge \tau} \frac{\partial^{2} \phi}{\partial r^{2}}(s, r) \sigma^{2}(s, r) d s=0 .
\end{gathered}
$$

Since $t$ is arbitrary, we have

$$
\frac{\partial \phi}{\partial t}+\gamma(t, r) \frac{\partial \phi}{\partial r}+\frac{1}{2} \sigma^{2}(t, r) \frac{\partial^{2} \phi}{\partial r^{2}}=0
$$

which governs the conditional probability of ruin $\phi(t, r)$ occurring between times $t$ and $T$ given the reserve $r$ at time $t$, where $0 \leq t \leq T<\infty$ and where $\gamma(t, r)$ defined by 
(1.6) is continuous but not differentiable at $d_{i}(t)$. The initial boundary conditions (2.2) follow by the definition of $\phi(t, r)$ in (2.1). We finished the proof.

We have now obtained a special class of a boundary value problem defined by a parabolic partial differential equation with a nonsmooth coefficient $\gamma(t, r)$ and a boundary condition being defined on the left half-opened space $r<0$ and a possibly vanishing diffusion coefficient $\sigma(t, r)$. The solution of such equations (2.13) and (2.2) will be discussed in Section 3. Before we close this section, we introduce the following theorem (see [11]) in the singular perturbation theory, which we will use later.

THEOREM 2.4. The parabolic internal layer solution $V$ caused by nonsmooth initial data $f(x)$ of the initial boundary value problem of the parabolic differential equation

$$
\begin{gathered}
\frac{\partial u}{\partial t}=p(t) \frac{\partial u}{\partial x}+\epsilon \frac{\partial^{2} u}{\partial x^{2}}, \quad u(0, x)=f(x), \quad-\infty<x<\infty \\
u(t, 0)=g(t), \quad 0<t<T
\end{gathered}
$$

is defined by

$$
V=v_{0}+O\left(\epsilon^{1 / 2}\right)
$$

where $P(t)=\int p(t)$ and

$$
v_{0}= \begin{cases}\frac{1}{2}[f](0) \operatorname{erfc}\left(\frac{x-P(t)}{2 \sqrt{t \epsilon}}\right), & x<P(t), \\ -\frac{1}{2}[f](0) \operatorname{erfc}\left(\frac{x-P(t)}{2 \sqrt{t \epsilon}}\right), & x>P(t),\end{cases}
$$

with $f(x)$ smooth everywhere except at $x=0$ with $[f](0)=f\left(0^{+}\right)-f\left(0^{-}\right)$,

$$
\lim _{x \rightarrow 0^{-}} f(x)=f\left(0^{-}\right), \quad \lim _{x \rightarrow 0^{+}} f(x)=f\left(0^{+}\right) .
$$

3. Asymptotic and numerical solutions of the diffusion equation for the probability of ruin. Consider the linear parabolic equation in (2.13) with the initial boundary conditions (2.2), where $0 \leq t \leq T \leq \infty$ and $\gamma(t, r)$ is as defined in (1.6) with $\sigma(t, r)$ and $\beta(t)$ defined as in (2.13). Let $\tau=T-t$ and make change of variables in (2.13) and (2.2), then we have

$$
\begin{gathered}
\frac{\partial \phi}{\partial \tau}=\gamma(\tau, r) \frac{\partial \phi}{\partial r}+\frac{1}{2} \sigma^{2}(\tau, r) \frac{\partial^{2} \phi}{\partial r^{2}}, \\
\phi(0, r)=0, \quad r>0, \\
\phi(\tau, r)=1, \quad r<0, \quad \lim _{r \rightarrow 0} \phi(t, \tau)=1,
\end{gathered}
$$

where $0 \leq \tau \leq T \leq \infty$ and

$$
\gamma(\tau, r)= \begin{cases}\pi(\tau)+\beta(\tau) r-\mu(\tau), & \text { if } r \leq d_{1}(\tau), \\ \pi(\tau)\left(1-\rho\left[\frac{r-d_{1}(\tau)}{d_{2}(\tau)-d_{1}(\tau)}\right]\right)+\beta(\tau) r-\mu(\tau), & \text { if } d_{1}(\tau)<r \leq d_{2}(\tau), \\ \pi(\tau)(1-\rho)+\beta(\tau) r-\mu(\tau), & \text { if } d_{2}(\tau)<r .\end{cases}
$$


Equations (3.1), (3.2), and (3.3) are an initial boundary value problem defined by a backward linear diffusion equation with nonsmooth convection coefficient $\gamma(\tau, r)$ defined in (3.4). It is clear that the probability of ruin $\phi(\tau, r) \equiv 1$ is defined on the left upper half-opened space $r<0$; we only need to consider the problem in the right upper space to determine the solution of (3.1) and (3.2) with the boundary condition

$$
\lim _{r \rightarrow 0} \phi(t, \tau)=1, \quad \tau>0 .
$$

We will divide the discussion into two cases: (I) $\sigma>k>0$ for some positive constant $k$ (nondegenerate case), (II) $\sigma \rightarrow 0^{+}$(the case of degenerate diffusion coefficient).

(I) $\sigma>k>0$ (nondegenerate case). The nonvanishing diffusion coefficient $\sigma>$ $k>0$ implies that a classical solution of (2.13) and (2.2) (or (3.1), (3.2), and (3.3)) exists and can be obtained using classical finite-difference scheme [12] for (2.13) and (2.2) defined by

$$
\phi_{k}^{n+1}=\phi_{k}^{n}+\gamma_{k}^{n} \delta_{0} \phi_{k}^{n} \frac{R}{2}+\alpha\left(\sigma^{2}\right)_{k}^{n} \delta^{2} \phi_{k}^{n},
$$

where $R=\Delta t / \Delta x, \delta_{0} \phi_{k}^{n}=\phi_{k+1}^{n}-\phi_{k-1}^{n}, \alpha=\Delta t / \Delta x^{2}$, and $\delta^{2} \phi_{k}^{n}=\phi_{k+1}^{n}-2 \phi_{k}^{n}+$ $\phi_{k-1}^{n}$. Even though the finite-difference mesh must be carefully set up to take care of initial boundary conditions (2.2) as well as to guarantee the stability and the convergence, it provides rather robust and accurate numerical scheme for (2.13) and (2.2) in this case. We will use some examples to illustrate the solution behavior of (2.13) and (2.2) for different dividend payments and interest rates.

EXAMPLE 3.1. Without loss of generality, assume that $\sigma(t, r)=e^{A(t)} \sigma_{0}$, where $\sigma_{0}$ is a positive constant. Let $\beta(t)=\beta e^{A(t)}$ with $\beta>0$ and $A(t)=\int_{0}^{t} \delta(s) d s$, where $\delta(t)$ acts as a functional force of inflation on claims and premiums. Let $\alpha=\pi(t)-\mu(t)$ and let $\gamma(t, r)$ be defined in (3.4). Then for time $T=10$, the space steps $k=500, \alpha=1$, $\beta=0.02$, and $\delta=\beta / 2$, we have the following.

(i) Dividend payments paid from the risk reserve. Figure 3.1 shows how the different dividend payments affect the behavior of the probability of ruin. We notice from this figure that the larger $\rho$, where $\rho$ is the portion of the premiums returned as dividends, the larger the probability of ruin $\phi(t, r)$, which makes sense.

(ii) Different dividend barriers. If we further provide different dividend barriers, Figure 3.2 shows how the probability of ruin is affected by the different dividend barriers. The figure shows that the larger the dividend barriers, the smaller the probability of ruin.

(iii) Different interest rates. Keep other parameters but the interest rates $\beta$ as in (i). Our numerical results show that the smaller the interest rate, the bigger the probability of ruin. See Figure 3.3.

(II) For the case $\sigma \rightarrow 0^{+},(3.1),(3.2)$, and (3.5),

$$
\begin{gathered}
\frac{\partial \phi}{\partial \tau}=\gamma(\tau, r) \frac{\partial \phi}{\partial r}+\frac{1}{2} \sigma^{2}(\tau, r) \frac{\partial^{2} \phi}{\partial r^{2}}, \\
\phi(0, r)=0, \quad r>0, \quad \lim _{r \rightarrow 0} \phi(\tau, r)=1, \quad \tau>0,
\end{gathered}
$$




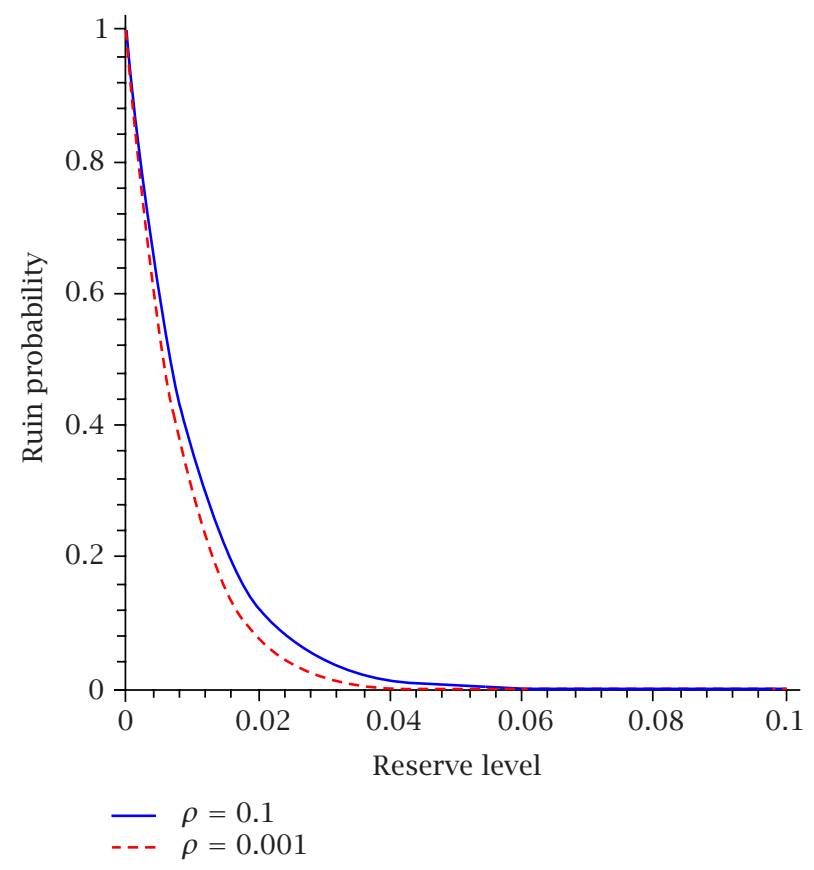

FIGURE 3.1. Probability of ruin when $\sigma$ is not small.

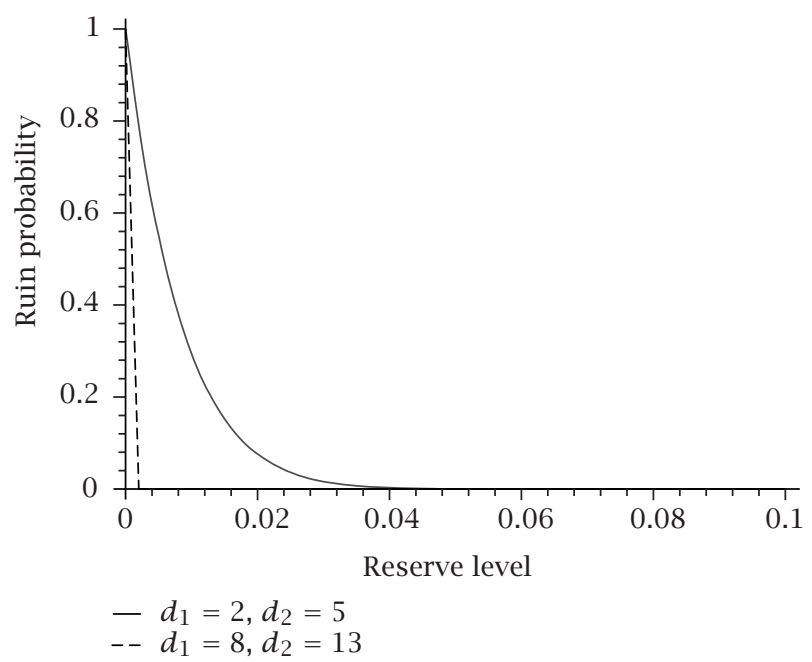

FIGURE 3.2. Probability of ruin when $\sigma$ is not small.

where $\gamma(\tau, r)$ is as defined in (3.4). We note that the initial boundary problem of (3.1), (3.2), and (3.5) is a singularly perturbed parabolic problem which can exhibit certain 


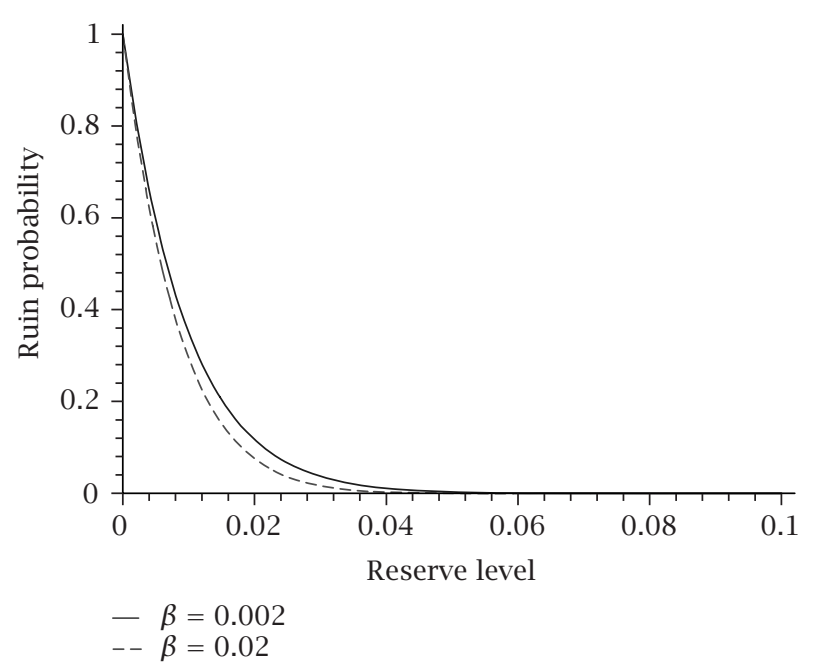

FIGURE 3.3. Probability of ruin when $\sigma$ is not small.

boundary layer, corner layer, or internal layers of rapid change in the solution and its derivatives; the imposition of initial boundary conditions is very subtle. We use the singular perturbation theory to determine the asymptotic behavior of the solution of (3.1), (3.2), and (3.4). Since the initial condition $\phi(0, r)=0$ for $r>0$ is homogeneous, there is no need for an initial layer near $\tau=0$ in this problem. It is known that the solution $\phi(\tau, r)$ of (3.1), (3.2), and (3.4) is strongly affected by the form of its convective coefficient $\gamma(t, r)$ which represents the total income flow. We notice that the claims rate $\mu(t)$ and the dividend payments only change the chance of ruin; we expect a chance to be there for ultimate ruin only when the rate at which premiums come in together with the investment income from the risk reserve is less than the average rate of claim together with the dividend payments, that is, $\gamma(\tau, r)<0$. We further divide our discussions into three different parameter regions depending on the total income flow $\gamma(\tau, r)$ of $(3.4)$ : (A) $\gamma(\tau, r)>0$, (B) $\gamma(\tau, r)<0$, and (C) $\gamma(\tau, r) \approx 0$.

We first consider the reduced equation of (3.1), which is a first-order hyperbolic equation with initial boundary conditions

$$
\begin{gathered}
\frac{\partial \phi_{0}}{\partial \tau}=\gamma(\tau, r) \frac{\partial \phi_{0}}{\partial r} \\
\phi_{0}(0, r)=0, \quad r>0, \quad \lim _{r \rightarrow 0} \phi_{0}(\tau, r)=1, \quad \tau>0 .
\end{gathered}
$$

CASE (A) $(\gamma(\tau, r)>0)$. In this case the characteristic curve $r=\Gamma\left(\tau ; r_{0}\right)=\Gamma(\tau)$ emanating from the point $\left(0, r_{0}\right)$, defined by the initial value problem

$$
\frac{d r}{d \tau}=-\gamma(\tau, r), \quad r(0)=r_{0}
$$

is assumed to exit on $\left[\tau_{0}, 0\right]$ with $\tau_{0}$ given by $\Gamma\left(\tau_{0} ; r_{0}\right)=0$. In other words, the characteristic curve $r=\Gamma\left(\tau ; r_{0}\right)$ exits at the point $\Gamma\left(\tau_{0}, 0\right)$. Therefore, the reduced solution 
$\phi_{0}(\tau, r)$ satisfies initial condition (3.2) and it does not agree with the given boundary data (3.5) near $r=0$. Thus the outer solution $\phi_{0}(\tau, r)$ is given by

$$
\phi_{0}=0, \quad r>0, \quad t>0 .
$$

A boundary layer of width $O\left(\sigma^{2}\right)$ is required along $r=0$ for $t>0$. It is in agreement with [14].

To obtain the boundary layer solution of (3.1), (3.2), and (3.5) for Case (A), without loss of generality, we let $O(\epsilon) \sim O(\sigma)$. Define the stretched variable $\xi$ along $r=0$ by

$$
\xi=\frac{r}{\sigma^{k}}
$$

insert (3.12) into (3.1), and evaluate the coefficient of $\partial \phi / \partial r$ at $r=0$. This gives, with $\psi=\psi(\tau, \xi)$,

$$
\frac{\partial \psi}{\partial \tau}=\sigma^{-k} \gamma(\tau, 0) \frac{\partial \psi}{\partial \xi}+\frac{1}{2} \sigma^{2} \sigma^{-2 k} \frac{\partial^{2} \psi}{\partial \xi^{2}} .
$$

Choose $k=2$ to balance the leading term; we have the boundary layer equation

$$
2 \sigma^{2} \frac{\partial \psi}{\partial \tau}=2 \gamma(\tau, 0) \frac{\partial \psi}{\partial \xi}+\frac{\partial^{2} \psi}{\partial \xi^{2}}
$$

where

$$
\gamma(\tau, 0)= \begin{cases}\pi(\tau)-\mu(\tau), & \text { if } 0 \leq d_{1}(\tau), \\ \pi(\tau)\left(1+\rho\left[\frac{d_{1}(\tau)}{d_{2}(\tau)-d_{1}(\tau)}\right]\right)-\mu(\tau), & \text { if } d_{1}(\tau)<0 \leq d_{2}(\tau), \\ \pi(\tau)(1-\rho)-\mu(\tau), & \text { if } d_{2}(\tau)<0 .\end{cases}
$$

It is clear that the dividend barriers $d_{i}(\tau), i=1,2$, at time $\tau$, are nonnegative by the definition and this implies that

$$
\gamma(\tau, 0)=\pi(\tau)-\mu(\tau) .
$$

It follows that $\gamma(\tau, 0)$ is differentiable with respect to $\tau$ since $\pi(\tau)$ and $\mu(\tau)$ are smooth functions. With solution $\psi$ expended in powers of $\epsilon$ with $\psi_{0}$ as its leading term, we find that

$$
\psi_{0}(\tau, \xi)=a(\tau)+b(\tau) e^{-2 \gamma(\tau, 0) \xi},
$$

where $a$ and $b$ are arbitrary functions to be determined using a matching condition and the boundary condition. It is clear that the case $\gamma(\tau, 0)>0$ implies that a parabolic boundary layer of width $O\left(\sigma^{2}\right)$ locates near $r=0$ by (3.17). Using the method of matching with a matching condition and the boundary conditions

$$
\begin{gathered}
\lim _{\xi \rightarrow 0} \psi(\tau, \xi)=\lim _{r \rightarrow 0} \phi(\tau, r)-\phi_{0}(\tau, 0)=1, \\
\lim _{\xi \rightarrow \infty} \psi(\tau, \xi)=0,
\end{gathered}
$$


we have the boundary layer solution $\psi(\tau, \xi)$ of width $O\left(\sigma^{2}\right)$ :

$$
\psi(\tau, \xi)=\psi_{0}(\tau, \xi)+O\left(\sigma^{2}\right)=e^{-2 \gamma(\tau, 0) \xi}+O\left(\sigma^{2}\right), \quad \xi>0 .
$$

To determine if there is a corner layer near origin $(0,0)$, we introduce a stretch variable $\varsigma$ along $\tau=0$, defined by

$$
\varsigma=\frac{\tau}{\sigma^{2}}
$$

in (3.13), then the corner layer solution $\varphi(\varsigma, \xi)$ is determined by a linear parabolic partial differential equation of the form

$$
\frac{\partial \varphi}{\partial \varsigma}=\gamma(0,0) \frac{\partial \varphi}{\partial \xi}+\frac{1}{2} \frac{\partial^{2} \varphi}{\partial \xi^{2}}
$$

for $0<\xi<\infty, 0<\varsigma<\infty$, subject to the initial boundary conditions

$$
\begin{gathered}
\varphi(0, \xi)=-\psi_{0}(0, \xi)=-e^{-2 \gamma(0,0) \xi}, \quad \xi>0, \\
\varphi(\varsigma, 0)=0, \quad \varsigma>0, \\
\lim _{\xi \rightarrow \infty} \varphi(\varsigma, \xi)=0, \quad \varsigma>0,
\end{gathered}
$$

where $\gamma(0,0)=\pi(0)-\mu(0)=$ constant by (3.16). Solving (3.21) and (3.22), we obtain the parabolic corner layer solution of width $O\left(\sigma^{2}\right)$,

$$
\varphi(\varsigma, \xi)=-e^{-2 \gamma(0,0) \xi-\gamma(0,0)^{2} \varsigma / 2} \operatorname{erfc}\left(\frac{\xi}{\sqrt{2 \varsigma}}\right),
$$

where the error function erfc is defined by

$$
\operatorname{erfc}(x)=\frac{2}{\sqrt{\pi}} \int_{0}^{x} e^{-s^{2}} d s .
$$

Therefore, we have the asymptotic solution of (3.1), (3.2), and (3.3) as $\sigma \rightarrow 0^{+}$and $\gamma(\tau, r)>0$, defined by

$$
\phi(\tau, r)=\phi_{0}(\tau, r)+\psi(\tau, \xi)+\varphi(\varsigma, \xi)+O\left(\sigma^{2}\right),
$$

where $\phi_{0}(\tau, r), \psi(\tau, \xi)$, and $\varphi(\varsigma, \xi)$ are defined by (3.11), (3.19), and (3.23).

CASE (B) $(\gamma(\tau, r)<0)$. In this case the outer solution $\phi_{0}(\tau, r)$ of (3.8) and (3.9) is significantly different from zero. When $\gamma(\tau, r)<0$, characteristic curve $r=\Gamma\left(\tau_{0} ; r\right)$ (see (3.10)) enters at the point $\Gamma\left(\tau_{0}, 0\right)$; the outer solution $\phi_{0}(\tau, r)$ must satisfy the given boundary condition of (3.3). Hence there is no boundary layer behavior in this case. This result is consistent with that obtained from (3.17), which shows that there is no boundary layer behavior in the neighborhood of $r=0$ when $\gamma(\tau, r) \leq 0$ since the exponential term decreases as $\xi$ decreases. On the other hand, in the sector between the characteristic curve $r>\Gamma(\tau)$ and the $r$-axis, the solution $\phi(\tau, r)$ of (3.1), (3.2), and (3.3) is small (approaching zero) as $\sigma \rightarrow 0^{+}$. In addition, there is an internal layer 
behavior caused by the nonsmooth initial data along the characteristic curve $r=\Gamma(\tau)$. Therefore, for $\gamma(\tau, r)<0$, we have an outer solution $\phi_{0}(\tau, r)$ supported by an internal layer solution $\varphi(\tau, \eta)$ of width $O(\sigma)$ along the characteristic curve $r=\Gamma(\tau)$,

$$
\phi(\tau, r)=\phi_{0}(\tau, r)+\varphi\left(\tau, \frac{r-\Gamma(\tau)}{\sigma}\right)+O(\sigma)
$$

where

$$
\phi_{0}(\tau, r)= \begin{cases}0, & \text { if } r<\Gamma(\tau), \\ 1, & \text { if } r>\Gamma(\tau),\end{cases}
$$

and $\Gamma(\tau)$ is defined by

$$
\Gamma(\tau)= \begin{cases}e^{\int-\beta(\tau) d \tau}\left(r_{0}+\int_{0}^{\tau} g_{1}(s) e^{\int_{0}^{s} \beta(u) d u} d s\right), & \text { if } r \leq d_{1}(\tau), \\ e^{\int b(\tau) d \tau}\left(r_{0}+\int_{0}^{\tau} g_{2}(s) e^{-\int_{0}^{s} b(u) d u} d s\right), & \text { if } d_{1}(\tau)<r \leq d_{2}(\tau), \\ e^{\int-\beta(\tau) d \tau}\left(r_{0}+\int_{0}^{\tau} g_{3}(s) e^{\int_{0}^{s} \beta(u) d u} d s\right), & \text { if } d_{2}(\tau)<r,\end{cases}
$$

where

$$
\begin{aligned}
& g_{1}(\tau)=\mu(\tau)-\pi(\tau), \quad g_{2}(\tau)=\frac{-\rho d_{1}(\tau) \pi(\tau)}{d_{2}(\tau)-d_{1}(\tau)}+g_{1}(\tau), \\
& g_{3}(\tau)=\mu(\tau)-\pi(\tau)(1-\rho), \quad b(\tau)=\frac{-\beta(\tau)+\rho \pi(\tau)}{d_{2}(\tau)-d_{1}(\tau)},
\end{aligned}
$$

obtained by (3.10). The internal layer solution $\varphi(\tau, \eta)$ can be obtained by introducing a sketch variable $\eta=(r-\Gamma(\tau)) / \sigma$ along the characteristic curve $r=\Gamma(\tau)$ and the similar techniques in Theorem 2.4 (see [11]). Thus $\varphi(\tau, \eta)$ has the following form:

$$
\varphi(\tau, \eta)= \begin{cases}\frac{1}{2} \operatorname{erfc}\left(\frac{\eta}{2 \sqrt{\tau}}\right)+O(\sigma), & r<\Gamma(\tau), \\ -\frac{1}{2} \operatorname{erfc}\left(\frac{\eta}{2 \sqrt{\tau}}\right)+O(\sigma), & r>\Gamma(\tau),\end{cases}
$$

where the complementary error function erfc is defined by

$$
\operatorname{erfc}(x)=\frac{2}{\pi} \int_{x}^{\infty} e^{-s^{2}} d s .
$$

CASE $(C)(\gamma(\tau, r) \approx 0)$. This is the case when the total income flow $\gamma(t, r) \approx 0$, in which we are assuming that the rate at which the business gains interest on its reserves together with the premiums-intake flow is about the same compared to the dividend payments and the average rate at which the claims arrived. Set $\gamma(\tau, r)=\sigma \Delta$, where $\Delta=O(1)$. Then we have

$$
\begin{gathered}
\frac{\partial \phi}{\partial \tau}=\sigma \Delta \frac{\partial \phi}{\partial r}+\frac{1}{2} \sigma^{2}(\tau, r) \frac{\partial^{2} \phi}{\partial r^{2}}, \\
\phi(0, r)=0, \quad r>0, \quad \lim _{r \rightarrow 0} \phi(\tau, r)=1, \quad \tau>0 .
\end{gathered}
$$


We know that the solution $\phi(\tau, r)$ of (3.1), (3.2), and (3.3) tends to the boundary condition

$$
\lim _{r \rightarrow 0} \phi(\tau, r)=1
$$

as $r \rightarrow 0$. Similarly, we expect that the solution $\phi(\tau, r)$ of (3.1), (3.2), and (3.3) tends to 1 as $\sigma \rightarrow 0^{+}$. Thus the asymptotic solution $\phi(\tau, r)$ of (3.32) with $\gamma(\tau, r) \approx 0$ as $\sigma \rightarrow 0^{+}$ has the form

$$
\begin{aligned}
\phi(\tau, r)=1-\frac{1}{2}( & 1-e^{(-2 r \Delta / \sigma)}+\operatorname{erfc}\left(\frac{(r+\sigma \tau \Delta)}{\sigma \sqrt{2 \tau}}\right) \\
& \left.-e^{(-2 r \Delta / \sigma)} \operatorname{erfc}\left(\frac{(\sigma \tau \Delta-r)}{\sigma \sqrt{2 \tau}}\right)\right)+O\left(\sigma^{2}\right),
\end{aligned}
$$

where $\Delta=O(1)$ and the error function erfc is defined in (3.24). Therefore, we have the following theorem.

THEOREM 3.2. Let $\sigma$ be a small positive parameter and let $0<t<T<\infty$.

(i) If the total income flow $\gamma(\tau, r)>0$, then as $\sigma \rightarrow 0^{+}$, the asymptotic solution of (2.13) and (2.2) has the following form:

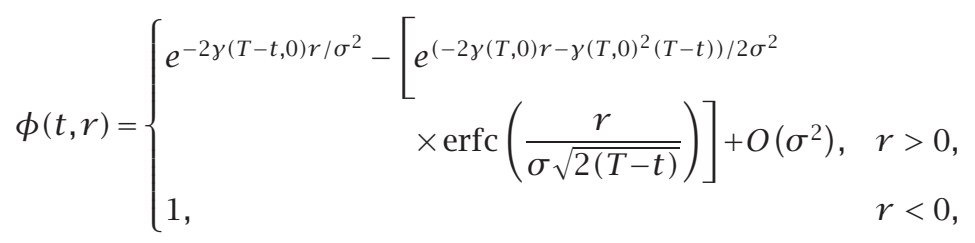

where $\gamma(0, T)=\pi(T)-\mu(T)$.

(ii) If the total income flow $\gamma(\tau, r)<0$, then as $\sigma \rightarrow 0^{+}$, the asymptotic solution of (2.13) and (2.2) has the following form:

$$
\phi(t, r)= \begin{cases}\phi_{0}(t, r)+\varphi\left(t, \frac{r-\Gamma(t)}{\sigma}\right)+O(\sigma), & r>0 \\ 1, & r<0\end{cases}
$$

where

$$
\phi_{0}(t, r)= \begin{cases}0, & \text { if } r<\Gamma(t) \\ 1, & \text { if } r>\Gamma(t)\end{cases}
$$

where $\Gamma(t)=\Gamma(T-\tau)=\Gamma(\tau)$ is defined as in (3.28) and the internal layer solution is defined by

$$
\varphi(t, r)= \begin{cases}\frac{1}{2} \operatorname{erfc}\left(\frac{r-\Gamma(t)}{2 \sigma \sqrt{T-t}}\right)+O(\sigma), & r<\Gamma(t), \\ -\frac{1}{2} \operatorname{erfc}\left(\frac{r-\Gamma(t)}{2 \sigma \sqrt{T-t}}\right)+O(\sigma), & r>\Gamma(t) .\end{cases}
$$




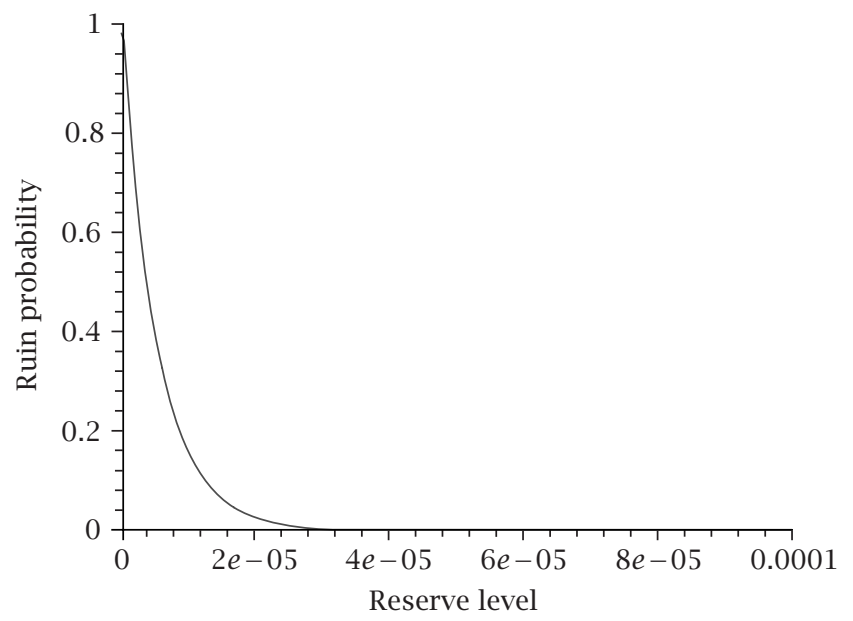

FIGURE 3.4. Probability of ruin when $\gamma>0$, time $=0.5$.

(iii) If the total income flow $\gamma(\tau, r) \approx \sigma \Delta \approx 0$, then as $\sigma \rightarrow 0^{+}$, the asymptotic solution of (2.13) and (2.2) has the following form:

$$
\phi(t, r)=\left\{\begin{aligned}
1-\frac{1}{2}\left(1-e^{(-2 r \Delta / \sigma)}+\operatorname{erfc}\left(\frac{(r+\sigma(T-t) \Delta)}{\sigma \sqrt{2(T-t)}}\right)\right. & \\
\left.-e^{(-2 r \Delta / \sigma)} \operatorname{erfc}\left(\frac{(\sigma(T-t) \Delta-r)}{\sigma \sqrt{2(T-t)}}\right)\right)+O\left(\sigma^{2}\right), & r>0, \\
1, & r<0 .
\end{aligned}\right.
$$

REMARK 3.3. For the case where $\sigma$ is not a small parameter or the case of a noise problem of (3.1) (in which we replace $\sigma$ by the noise coefficient $\sigma \epsilon$ with $\epsilon \rightarrow 0^{+}$), we can obtain a similar result using the above techniques.

We now use the following examples to illustrate the results of Theorem 3.2.

EXAMPLES. To illustrate our results of Theorem 3.2, we choose the following parameters: $\sigma=0.005, T=10, \rho=0.01, \beta(\tau)=\beta_{0} e^{\tau}, \beta_{0}=0.01$, and $r_{0}=10$.

For Case (A) Figure 3.4 shows the probability of ruin when $\pi=25, \mu=24, d_{1}=2$, and $d_{2}=5$. A 3D figure (Figure 3.5 ) shows the boundary layer behavior.

For Case (B), we first show an example of $\gamma(t, r)$ function which is negative when $\pi=20, \mu=25, d_{1}=9$, and $d_{2}=15$ (see Figure 3.6). Figure 3.7 shows the internal layer behavior of the probability of ruin.

For Case (C), we first show the case $\gamma(t, r)=0$ when $\Delta=1$ (see Figure 3.8). Figures 3.9 and 3.10 show the behavior of ruin probability at which $\gamma(t, r) \rightarrow 0$.

REMARK 3.4. When $\sigma \rightarrow 0^{+}$and the total income flow $\gamma(\tau, r)$ changes signs, the solution behavior of (3.1), (3.2), and (3.3) becomes much more complicated. Consider the characteristic curve $r=\Gamma\left(\tau ; r_{0}\right)=\Gamma(\tau)$ as in (3.10). If $\gamma(\tau, r)>0$ for all $\tau>\tau_{m}$, then $\left(\tau_{m}, r_{m}\right) \in(0, T) \times(0, \infty)$ such that $\gamma\left(\tau_{m}, r_{m}\right)=0$. The asymptotic solution of (3.1), 


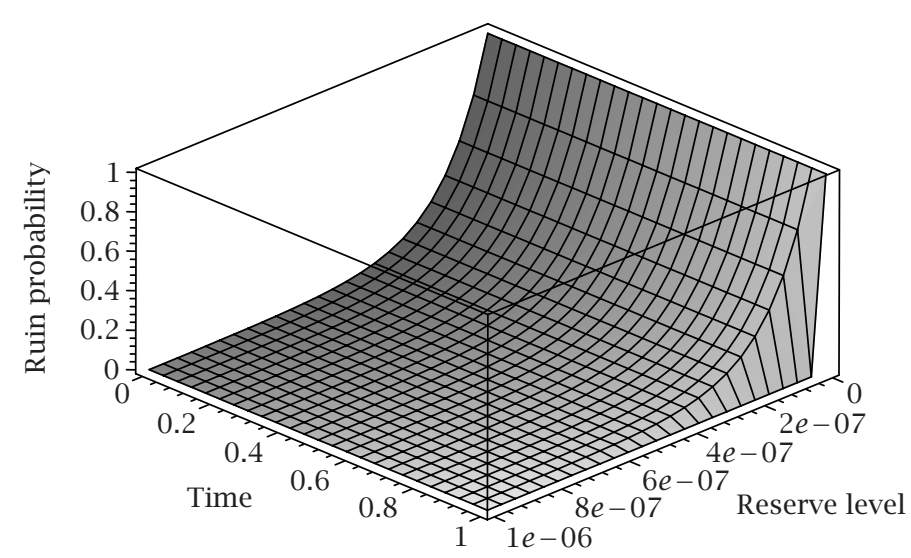

FIGURE 3.5. Probability of ruin when the total income flow is positive.

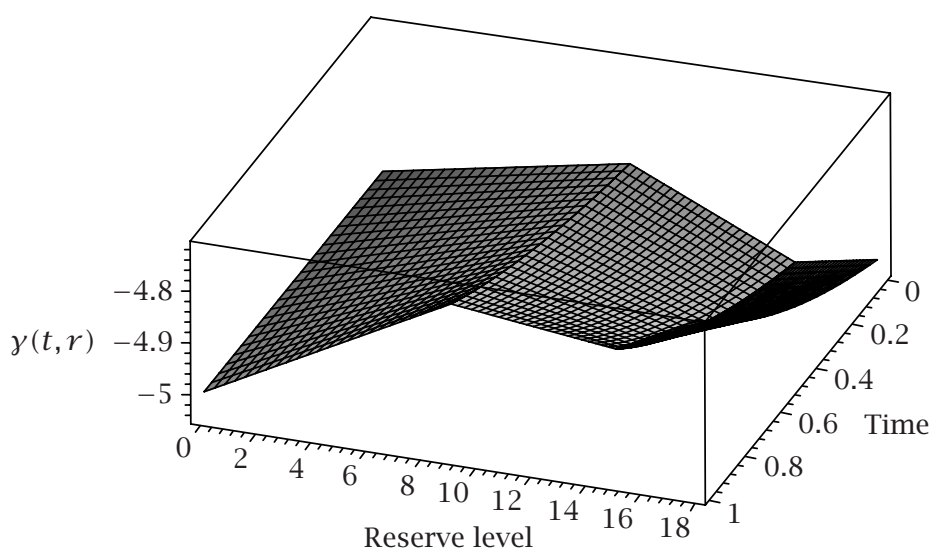

FIGURE 3.6. Total income flow is negative

(3.2), and (3.3) has boundary layer behavior near the neighborhood of $r=0$, where we first solve the associate reduced equation (3.8) numerically with the initial condition $\phi(0, r)=0$ for all $\tau<\tau_{m}$ and $r<r_{m}$ supported by a boundary layer solution and a corner layer solution both of order $O\left(\sigma^{2}\right)$. On the other hand, when $\gamma(\tau, r)<0$ for all $\tau<\tau_{m}$, the outer solution must satisfy both initial boundary conditions and there is an internal layer of order $O(\sigma)$ along the characteristic curve $r=\Gamma(\tau)$ for all $\tau<\tau_{m}$. We can extend the idea to the case with multiple turning points. An asymptotic guided numerical method can be used to provide an accurate numerical solution of (2.13) and (2.2) as $\sigma \rightarrow 0$. To avoid stuffing this paper, this case will be discussed elsewhere.

4. Conclusion and remarks. We studied the diffusion models for compounded risk reserves (1.1) and (1.3) with dividend payments. For the case when dividend payments 


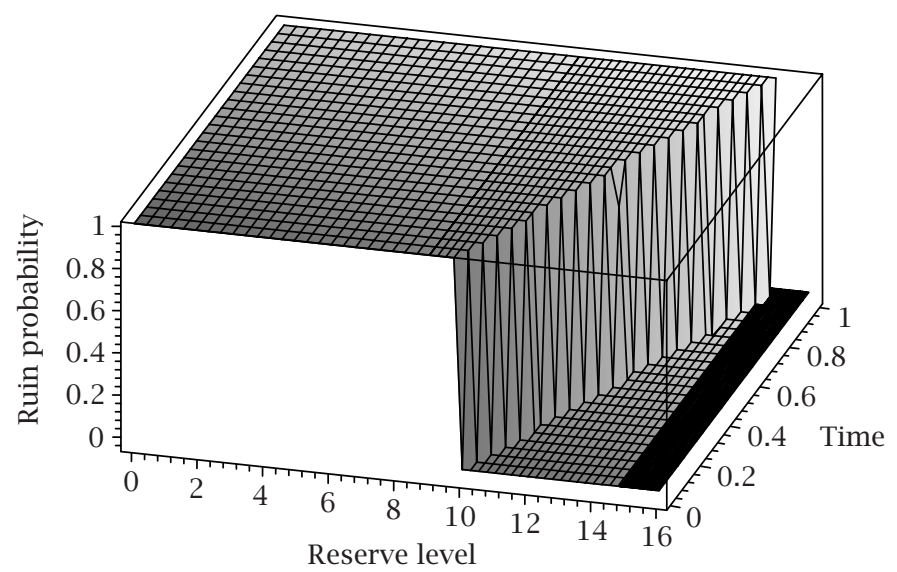

FIGURE 3.7. Internal layer solution along the characteristic curve $r=\Gamma(t, r)$.

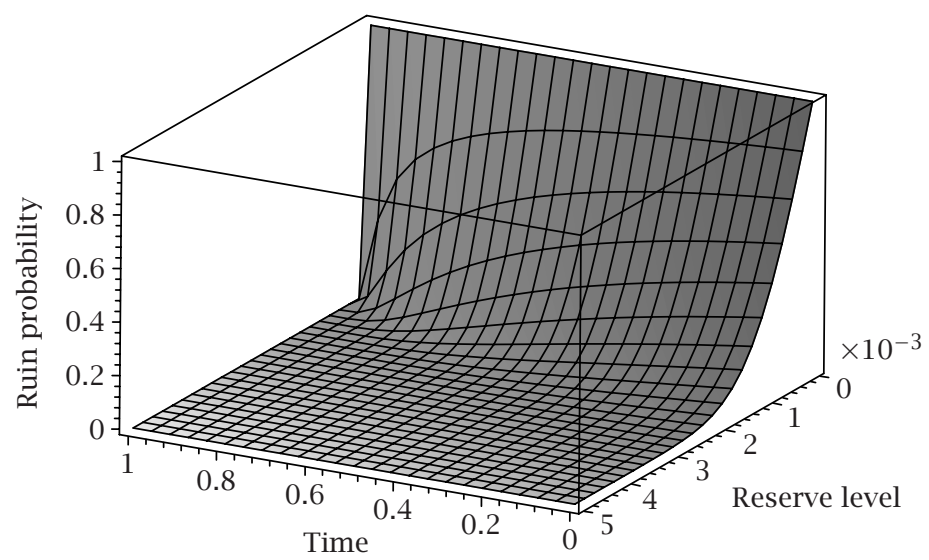

FIGURE 3.8. Case (C). Ruin probability when $\gamma(t, r)=0$.

are paid from the risk reserves, using stochastic calculus and martingale theory, we obtained a special class of a boundary value problem defined by a parabolic partial differential equation with a nonsmooth coefficient $\gamma(t, r)$ and a boundary condition being defined on the left half-opened space $r<0$ and a possibly degenerate diffusion coefficient $\sigma(t, r)$. According to the different behavior of the total income flow $\gamma(t, r)$ and the diffusion coefficient $\sigma(t, r)$, numerical and asymptotic solutions of probability of ruin over finite time are obtained using numerical scheme (3.6) and asymptotic analysis. Our examples show that when the diffusion coefficient $\sigma$ is not small, the probability of ruin increases as dividend payments increase; it decreases as dividend barriers increase. For the same dividend payments, the interest rate also plays a role in affecting the solution behavior that probability of ruin decreases as the interest rate increases. 


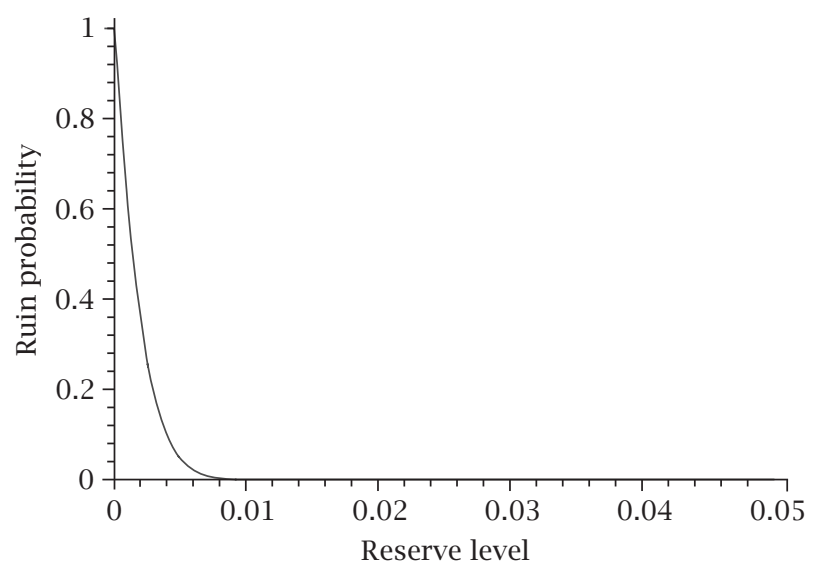

FIGURE 3.9. Probability of ruin when $\gamma \rightarrow 0$, time $=0.5$.

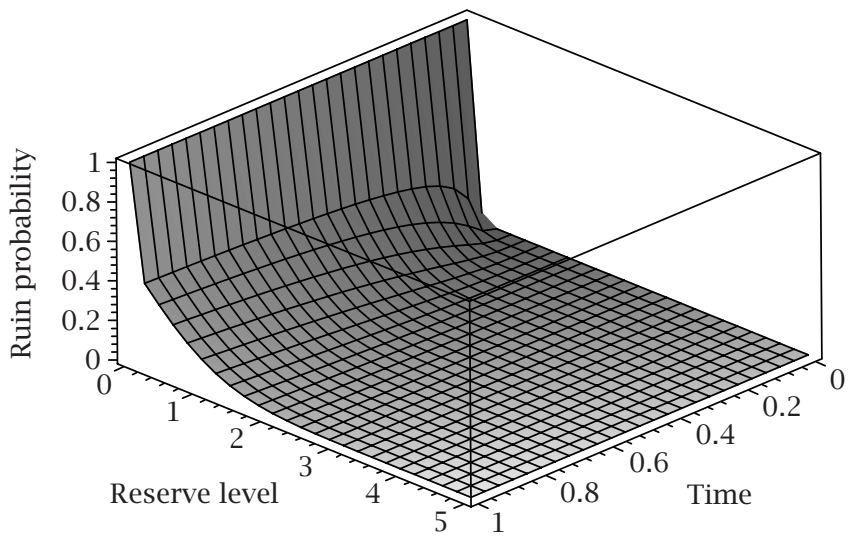

FIGURE 3.10. Probability of ruin when $\gamma \rightarrow 0$

For the case of diffusion coefficient $\sigma \rightarrow 0^{+}$, the solution behavior is quite different based on the signs of the total income flow $\gamma(t, r)$. When the premium flow, together with the investment income from the risk reserve, is greater than the average claim flow, together with the dividend payments if there are any, we have the total income flow $\gamma(t, r)>0$ and the probability of ruin $\phi(t, r)$ is quite small and it is not affected by the dividend payments as long as the total income flow keeps increasing. On the contrary, when the total income flow $\gamma(t, r)<0$, the ruin occurs along the reserve level $r<\Gamma(t, r)$ with a rate $-\gamma(t, r)$ (see Case (B)) then dropping to zero when $r<\Gamma(t, r)$. We can see from the examples that the initial reserve level and the dividend payments play critical roles in this case. When $\gamma(\tau, r) \approx 0$, this is the case when the total income flow $\gamma(t, r) \approx 0$, in which we are assuming that the rate at which the business gains interest on its reserves together with the premiums-intake flow is about the same compared to 
the dividend payments and the average rate at which the claims arrived. The probability of ruin $\phi(t, r)$ is much larger in this case, but still within thin layer compared with Case (B). The dividend payment plays a little role in this case. These results make sense since the claims rate $\mu(t)$ and the dividend payments only change the chance of ruin; we expect a chance to be there for ultimate ruin only when the rate at which premiums come in together with the investment income from the risk reserve is less than the average rate of claim together with the dividend payments. These results can be extended to a higher dimension in which the multiple portfolios of risk reserves are considered. We are currently investigating such an extension with the turning-point case.

\section{REFERENCES}

[1] R. J. Elliott, Stochastic Calculus and Applications, Applications of Mathematics, vol. 18, Springer-Verlag, Berlin, 1982.

[2] W. H. Fleming and H. M. Soner, Controlled Markov Processes and Viscosity Solutions, Applications of Mathematics, vol. 25, Springer-Verlag, New York, 1993.

[3] M. Freidlin, Functional Integration and Partial Differential Equations, Annals of Mathematics Studies, vol. 109, Princeton University Press, New Jersey, 1985.

[4] J. Garrido, Diffusion premiums for claim severities subject to inflation, Insurance Math. Econom. 7 (1988), no. 2, 123-129.

[5] - Stochastic differential equations for compounded risk reserves, Insurance Math. Econom. 8 (1989), no. 3, 165-173.

[6] I. I. Gīhman and A. V. Skorohod, Stochastic Differential Equations, Springer-Verlag, Berlin, 1972.

[7] J. Grandell, Aspects of Risk Theory, Springer Series in Statistics: Probability and Its Applications, Springer-Verlag, New York, 1991.

[8] C. M. Møller, Stochastic differential equations for ruin probabilities, J. Appl. Probab. 32 (1995), no. 1, 74-89.

[9] M. Musiela and M. Rutkowski, Martingale Methods in Financial Modelling, Applications of Mathematics, vol. 36, Springer-Verlag, Berlin, 1997.

[10] S. Shao, Asymptotic solutions of diffusion models for risk reserves, Int. J. Math. Math. Sci. 2003 (2003), no. 35, 2221-2239.

[11] S.-D. Shih, On a class of singularly perturbed parabolic equations, ZAMM Z. Angew. Math. Mech. 81 (2001), no. 5, 337-345.

[12] G. D. Smith, Numerical Solution of Partial Differential Equations: Finite Difference Methods, 3rd ed., Oxford Applied Mathematics and Computing Science Series, The Clarendon Press, Oxford University Press, New York, 1985.

[13] V. R. Young and T. Zariphopoulou, Computation of distorted probabilities for diffusion processes via stochastic control methods, Insurance Math. Econom. 27 (2000), no. 1, $1-18$.

[14] E. Zauderer, Partial Differential Equations of Applied Mathematics, Pure and Applied Mathematics, John Wiley \& Sons, New York, 1983.

S. Shao: Department of Mathematics, Cleveland State University, Cleveland, OH 44115, USA

E-mail address: s.shao@csuohio.edu

C. L. Chang: Department of Mathematics, Cleveland State University, Cleveland, OH 44115, USA

E-mail address: c. chang@csuohio.edu 


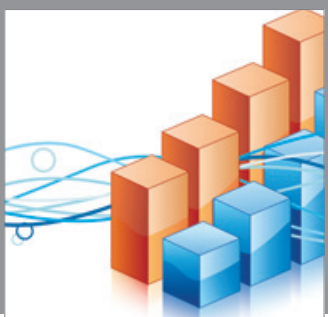

Advances in

Operations Research

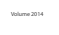

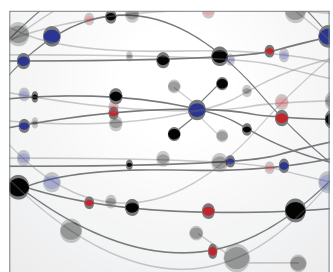

\section{The Scientific} World Journal
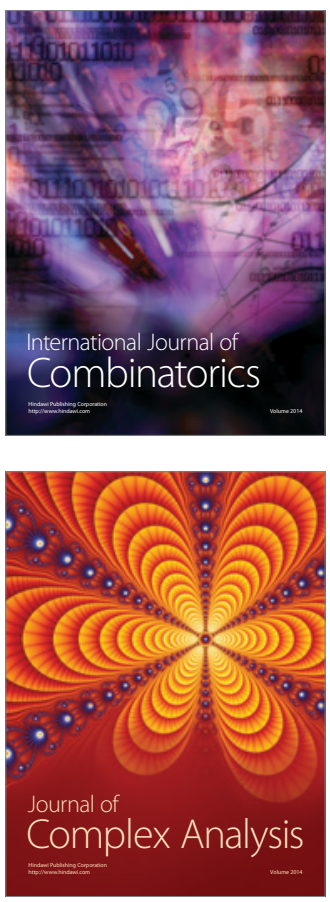

International Journal of

Mathematics and

Mathematical

Sciences
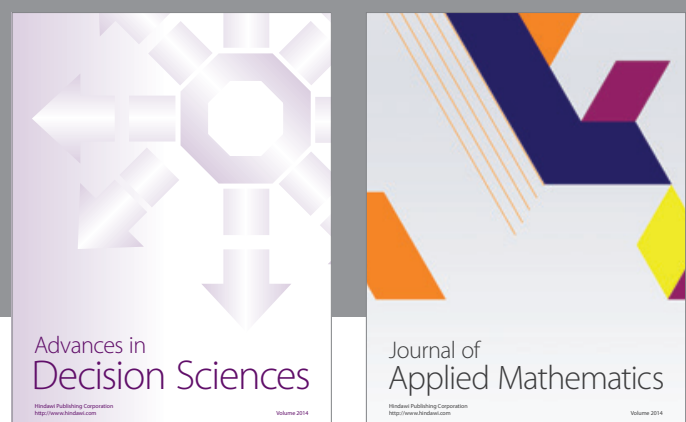

Journal of

Applied Mathematics
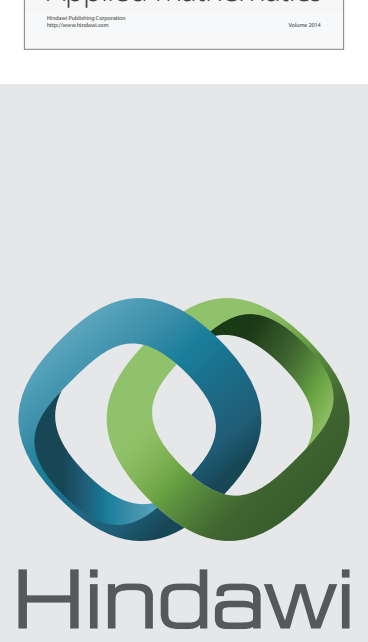

Submit your manuscripts at http://www.hindawi.com
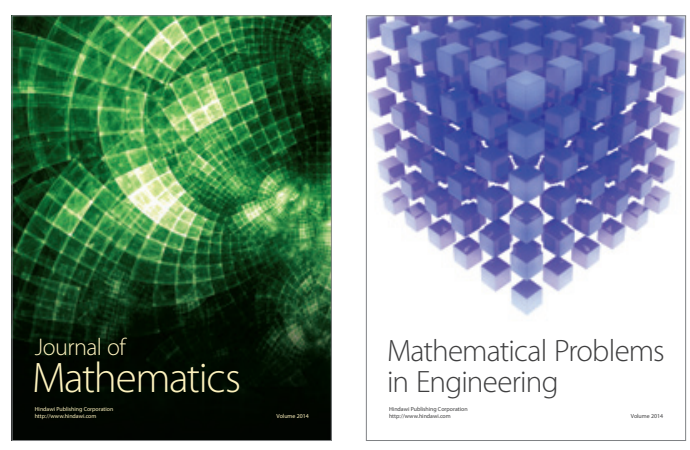

Mathematical Problems in Engineering
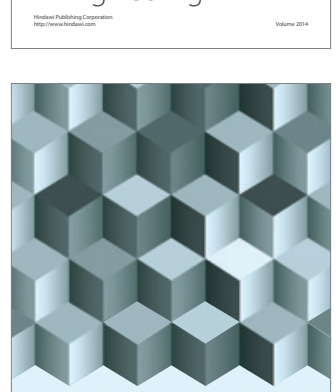

Journal of

Function Spaces
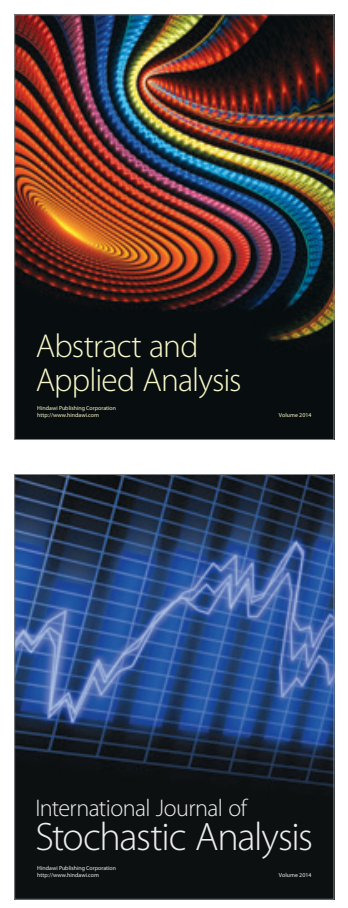

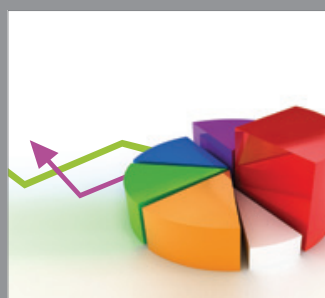

ournal of

Probability and Statistics

Promensencen
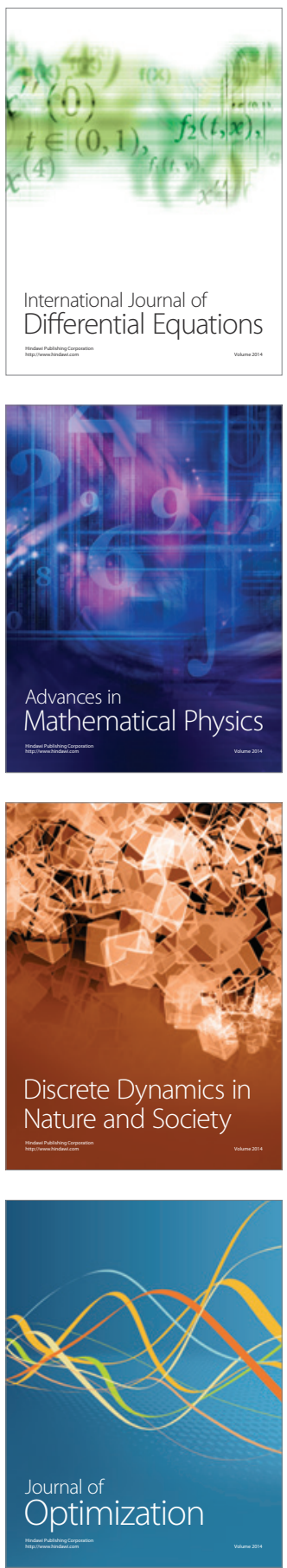